\title{
Photonics of Quantum-Dot Nanomaterials and Devices
}

THEORY AND MODELLING 
This page intentionally left blank 


\title{
Photonics of \\ Quantum-Dot \\ Nanomaterials \\ and Devices \\ THEORY AND MODELLING
}

\author{
ORTWIN HESS \\ Imperial College London, UK \\ EDELTRAUD GEHRIG \\ Hochschule Heilbronn, Germany
}

Imperial College Press 
Published by

Imperial College Press

57 Shelton Street

Covent Garden

London WC2H 9HE

\section{Distributed by}

World Scientific Publishing Co. Pte. Ltd.

5 Toh Tuck Link, Singapore 596224

USA office: 27 Warren Street, Suite 401-402, Hackensack, NJ 07601

UK office: 57 Shelton Street, Covent Garden, London WC2H 9HE

\section{British Library Cataloguing-in-Publication Data}

A catalogue record for this book is available from the British Library.

\section{PHOTONICS OF QUANTUM-DOT NANOMATERIALS AND DEVICES Theory and Modelling}

Copyright (C) 2012 by Imperial College Press

All rights reserved. This book, or parts thereof, may not be reproduced in any form or by any means, electronic or mechanical, including photocopying, recording or any information storage and retrieval system now known or to be invented, without written permission from the Publisher.

For photocopying of material in this volume, please pay a copying fee through the Copyright Clearance Center, Inc., 222 Rosewood Drive, Danvers, MA 01923, USA. In this case permission to photocopy is not required from the publisher.

ISBN-13 978-1-84816-521-2

ISBN-10 1-84816-521-8

Typeset by Stallion Press

Email: enquiries@stallionpress.com

Printed in Singapore. 


\section{To Jens, Rebecca and Gabriel And}

To Heike, Rita, Francis, Killian and Maxwell 
This page intentionally left blank 


\section{Contents}

1. Introduction to Photonic Quantum Dot

Nanomaterials and Devices 1

1.1 Physical Properties of Quantum Dots . . . . . . . 1

1.2 Active Semiconductor Gain Media . . . . . . . . . 2

1.3 Quantum Dot Lasers . . . . . . . . . . . . . . 4

1.3.1 Heterostructure lasers . . . . . . . . . . . 4

1.3.2 Active nanomaterials . . . . . . . . 5

1.4 Laser Cavities . . . . . . . . . . . . . . . . 6

1.4.1 In-plane edge-emitting lasers . . . . . . . . 7

1.4.2 Vertical-cavity surface-emitting lasers . . . . 8

1.4.3 High-power laser amplifiers . . . . . . . . . . 9

1.4.4 Coupled-cavity systems . . . . . . . . . . 10

1.4.5 Optically excited nano systems . . . . . . . 11

1.4.6 QD metastructures ........... 11

References .................... 12

2. Theory of Quantum Dot Light-Matter Dynamics 15

2.1 Rate Equations . . . . . . . . . . . . . . . 19

2.2 Maxwell-Bloch Equations . . . . . . . . . . . . 24

2.2.1 Mesoscopic two-level approach . . . . . . . 25

2.2.2 Mesoscopic Maxwell-Bloch description of multi-level quantum dot systems . . . . . 29

2.2.2.1 Optical field dynamics . . . . . . 30 


\subsubsection{Carrier dynamics within}

a quantum dot . . . . . . . . 34

2.3 Quantum Luminescence Equations . . . . . . . . . . . 38

2.4 Quantum Theoretical Description . . . . . . . . . 42

References .................. 43

3. Light Meets Matter I: Microscopic Carrier Effects and Fundamental Light-Matter Interaction 45

3.1 Dynamics in the Active Charge Carrier Plasma . . . 46

3.1.1 Intra-dot carrier scattering . . . . . . . . 47

3.1.2 Phonon induced carrier scattering between quantum dots and wetting layer . . . . . . 48

3.1.3 Auger scattering processes involving 0D and $2 \mathrm{D}$ carriers . . . . . . . . . . . . 49

3.1.4 Level and gain dynamics . . . . . . . . . 51

3.1.5 Dynamics of carrier scattering rates . . . . . 54

3.2 Dynamic Level Hole Burning . . . . . . . . . . . 57

3.3 Ultrashort Nonlinear Gain and Index Dynamics . . . 62

3.4 Conclusion . . . . . . . . . . . . . . 69

References ................... 69

4. Light Meets Matter II: Mesoscopic Space-Time

Dynamics

4.1 Introduction: Transverse and Longitudinal Mode Dynamics . . . . . . . . . . . . . 71

4.2 Influence of the Transverse Degree of Freedom and Nano-Structuring on Nearfield Dynamics and Spectra . . . . . . . . . . . . . . 72

4.3 Longitudinal Modes . . . . . . . . . . . . . . 77

4.4 Coupled Space-Time Dynamics in the Active Area . . . . . . . . . . . . . . . 78

4.4.1 Influence of injection level and geometry . . 79

4.4.2 Influence of disorder: the spatially inhomogeneous quantum dot ensemble . . . 83 
4.4.3 Light fluctuations and mode competition in quantum dot cavities . . . . . . . . . 86

4.5 Conclusion . . . . . . . . . . . . . 97 References ................. 97

5. Performance and Characterisation: Properties on Large Time and Length Scales

5.1 Introduction . . . . . . . . . . . . . . . . . . . 101

5.2 Spatial and Spectral Beam Quality . . . . . . . . 102

5.3 Dynamic Amplitude Phase Coupling . . . . . . . . 105

5.4 Conclusion . . . . . . . . . . . . . . 113

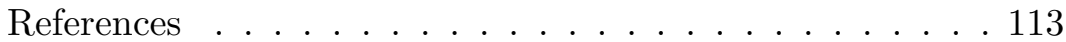

6. Nonlinear Pulse Propagation in Semiconductor Quantum Dot Lasers

6.1 Dynamic Shaping of Short Optical Pulses . . . . . . 116

6.2 Nonlinear Femtosecond Dynamics of Ultrashort Light Pulses . . . . . . . . . . . . 118 6.2.1 Self-induced propagation control: tunable propagation speed . . . . . . . . 118 6.2.2 Propagation control by a second pulse . . . . 123 6.3 Conclusion . . . . . . . . . . . . . 125 References ..................... 125

$\begin{array}{ll}\text { 7. High-Speed Dynamics } & 127\end{array}$

7.1 Mode-Locking in Multi-Section Quantum Dot Lasers . . . . . . . . . . . . . . . . . 127

7.2 Dependence of Pulse Duration on Injection Current, Bias Voltage and Device Geometry . . . . . . . . 129

7.3 Radio Frequency Spectra of the Emitted Light . . . 132

7.4 Short-Pulse Optimisation . . . . . . . . . . 134

7.5 Conclusion . . . . . . . . . . . . 136

References ..................... 137 
8. Quantum Dot Random Lasers

8.1 Spatially Inhomogeneous Semiconductor

Quantum Dot Ensembles . . . . . . . . . . . . 139

8.1.1 Gain spectra . . . . . . . . . . . . . 141

8.1.2 Spatial and spectral hole burning . . . . . . 142

8.2 Coherence Properties . . . . . . . . . . . . . . 145

8.3 Random Lasing in Semiconductor Quantum

Dot Ensembles . . . . . . . . . . . . . 150

8.3.1 The physics of random lasing . . . . . . 150

8.3.2 Lasers with strong disorder: incoherent feedback . . . . . . . . . . . 152

8.3.3 Lasers with weak disorder: coherent feedback . . . . . . . . . 155

8.4 Conclusion . . . . . . . . . . . . . 157

References ..................... 157

9. Coherence Properties of Quantum Dot

Micro-Cavity Lasers

9.1 Introduction . . . . . . . . . . . . . . . . 159

9.2 Radial Signal Propagation and Coherence

Trapping . . . . . . . . . . . . . . 161

9.3 Influence of Disorder . . . . . . . . . . . . . . . . . . . . . . . . . . . . . . .

9.4 Conclusions . . . . . . . . . . . . . . . . 170

References ..................... 170 